\title{
The Genus Melodinus (Apocynaceae): Chemical and Pharmacological Perspectives
}

\author{
Yao Lu*, T. J. Khoo, C. Wiart \\ School of Pharmacy, Faculty of Science, University of Nottingham (Malaysia Campus), \\ Semenyih, Malaysia \\ Email: "emilyly@126.com, ${ }^{*}$ khyx2lyo@nottingham.edu.my
}

Received 2 April 2014; revised 5 May 2014; accepted 22 May 2014

Copyright (C) 2014 by authors and Scientific Research Publishing Inc.

This work is licensed under the Creative Commons Attribution International License (CC BY). http://creativecommons.org/licenses/by/4.0/

\begin{abstract}
The plants of the genus Melodinus (Apocynaceae) are widely distributed, and have long been used in folk medicine for the treatment of various ailments such as meningitis in children and rheumatic heart diseases, hernia, infantile malnutrition, dyspepsia and testitis. Over 100 alkaloids together with flavonoids, lignans, steroids, terpenoids and coumarins have been identified in the genus, and many of these have been evaluated for biological activity. This review presents comprehensive information on the chemistry and pharmacology of the genus together with the traditional uses of many of its plants. In addition, this review discusses the structure-activity relationship of different compounds as well as recent developments and the scope for future research in this aspect.
\end{abstract}

Keywords

Melodinus, Apocynaceae, Ethnopharmacology, Anti-Cancer

\section{Introduction}

\subsection{General Introduction of the Family Apocynaceae Juss. and Genus Melodinus}

The genus Melodinus belongs to family Apocynaceae, a large family of a family of flowering plants that includes trees, shrubs, herbs, stem succulents, and vines, commonly called the dogbane family [1]. The family comprises some 1500 species divided over about 424 genera. The former family Asclepiadaceae is included in Apocynaceae according to the APG III system [2]. Members of the family are native to European, Asian, African, Australian and American tropics or subtropics, with some temperate members [1]. Many species are tall

*Corresponding author. 
trees found in tropical rainforests, but some grow in tropical dry (xeric) environments [3]. There are also perennial herbs from temperate zones [4]. Some are sources of important drugs, such as cardiac glycosides, which affect heart function [5]. These include the Acokanthera, Apocynum, Cerbera, Nerium, Thevetia and Strophantus. Rauvolfia serpentina, or Indian Snakeroot, yields the alkaloids reserpine and rescinnamine, which are useful tools in the treatment of high blood pressure and even some forms of psychosis. Catharanthus roseus yields alkaloids used in treating cancer [6] [7].

Melodinus clade embraces 109 species which are mainly found in tropical Asia and Oceania [8].

\subsection{Botanical Features of Melodinus}

The members of the genus Melodinus consist of stout climber or lianas. Branches dark gray, glabrous; young branch lets and leaves scaly. Petiole $5 \mathrm{~mm}-10 \mathrm{~mm}$; leaf blade oblong or narrowly elliptic, $7 \mathrm{~cm}-18 \mathrm{~cm} \times 2.5$ $\mathrm{cm}-5.2 \mathrm{~cm}$, papery, base rounded, apex acuminate; lateral veins $10-15$ pairs, nearly flat on both surfaces. Cymes umbellate, terminal and axillary, $5 \mathrm{~cm}-6.5 \mathrm{~cm}$; peduncle $1.5 \mathrm{~cm}-2 \mathrm{~cm}$, glabrous; bracts and bracteoles $3 \mathrm{~mm}-7 \mathrm{~mm}$. Pedicel $5 \mathrm{~mm}-7 \mathrm{~mm}$, pubescent. Flower buds cylindric, ca. $2 \mathrm{~cm}$, glabrous outside. Sepals broadly ovate, ca. $75 \mathrm{~mm}$, ciliate, apex acute. Corolla white, tube ca. $1.2 \mathrm{~cm}$, pubescent inside; lobes oblong, ca. $1.1 \mathrm{~cm}$; corona scales linear, decurrent to lower part of corolla tube, included. Ovary glabrous. Style very short. Berries globose, ca. $10.5 \mathrm{~cm}$ in diam [9]-[11].

This study is an attempt to compile an up-to-date and comprehensive review of the genus Stephania that covers its traditional medicinal uses, chemistry and pharmacology. Many plants of this genus are pharmacologically known but chemically unknown and vice-versa. Therefore, the scope of future research in this aspect is also discussed here.

\section{Traditional and Medicinal Uses}

Melodinus suaveolens and Melodinus henryi, have been used in Chinese folk medicine for the treatment of meningitis in children and rheumatic heart diseases [11]. They can also "invigorate the circulation of blood", "stimulate sucking and treat fracture" [12]. It was also used in Chinese folk medicine for the treatment of hernia, infantile malnutrition, dyspepsia and testitis [11].

Melodinus reticulatus was used to treat cancers as alkaloids were detected in 1983 [13]. Next, several alkalois were detected successively from Melodinus acutiflorus and Melodinus tenuicaudatus [14] [15].

\section{Chemical Constituents}

Isolation and structure elucidations of secondary metabolites in Melodinus have been carried out since the 80s [16]. Majority of investigations include the acrial part of the species. To date about 100 compounds have been isolated from 12 species, which are Melodinus acutiflorus F. Muell, Melodinus aeneus Baill, Melodinus balansae Baill, Melodinus celastroides Baill, Melodinus fusiformis Champ. ex Benth, Melodinus guillauminii Boiteau, Melodinus hemsleyanus Diels, Melodinus morsei Tsiang, Melodinus monogynus Roxb, Melodinus scandens J.R. Forst. \& G. Forst., Melodinus suaveolens (Hance) Champ. ex Benth, Melodinus yunnanensis Tsiang \& P.T. Li.

The isolation and separation technique is very much dependent on the type of fractions. Substances are separated with liquid chromatography using different solvent mixtures with silica gel [13], charcoal [17] and sephadex [14] [18]. Other types of analytical techniques include thin layer chromatography (TLC) and high performance liquid chromatography (HPLC) [19]-[22].

The structures are mainly established by mass spectroscopy (MS), ultra-violet spectroscopy (UV), infrared spectroscopy (IR) and ${ }^{1} \mathrm{H}$ and/or ${ }^{13} \mathrm{C}$ nuclear magnetic resonance (NMR). ${ }^{1} \mathrm{H}$ and/or ${ }^{13} \mathrm{C}$ spectroscopy is probably the most useful method in structure elucidation [13]-[15] [17]-[20] [22]-[30].

Among the secondary metabolites isolated from members of the genus Melodinus are mainly focused on alkaloids (indoles, steroids, diterpenes). The main secondary metabolites isolated so far from the genus Melodinus. consists of 30 - 35 compounds. The profile of all known secondary metabolites of Melodinus as found in literature and their structures are included following.

\subsection{Melodinus acutiflorus F. Muell}

Rhaxicine 1), rhaximine 2), 16-epi rhazinaline 3), N(l)-methyl-rhazicine 4) [14] [32]. 


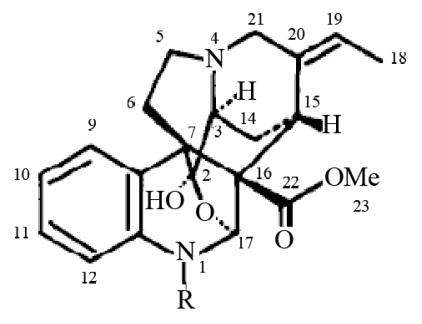

$1 \mathrm{R}=\mathrm{H}$,

$4 \mathrm{R}=\mathrm{Me}$

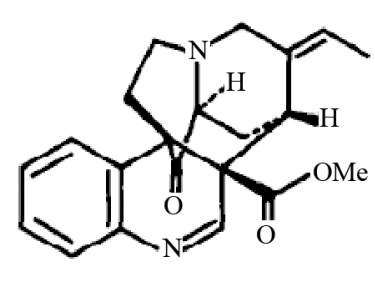

2 Rhazlmine

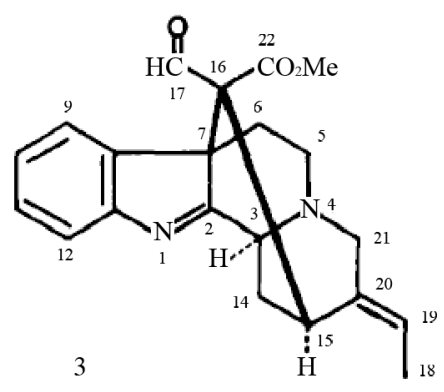

16-epi-rhazinallne

\subsection{Melodinus aeneus Baill}

$(+)$ vincadifformine (5), $(-)$ tabersonine (6), (-) methoxy-11 tabersonine (7), $(-)$ lochnericine (8), $(-)$ lochneinine (9), (-) ibogamine (10), venoterpine (11), (+) tubotaiwine (12), $\left(^{+}\right)$N-oxy tubotaiwine (13), $(+)$epi16dehydro l4-15 vincamine (14), (+) epi-16dehydro 14-15 vincine (15) [33].<smiles>CCC12CCCN3CCC4(C(=C(C(C)=O)C1)Nc1ccccc14)C32</smiles><smiles>CCC1C2CCC1C1CC2CCc2c1[nH]c1ccccc21</smiles>

10<smiles></smiles>

$\begin{array}{ll}5 \quad \mathrm{R} & =\mathrm{H} \\ 7 & \mathrm{R}\end{array}$<smiles>CC1c2cnccc2CC1O</smiles><smiles>[R]c1ccc2c(c1)NC1=C(C(=O)OC)CC3(CC)C4OC4CN3CCC12C</smiles>

$8 \mathrm{R}=\mathrm{H}$

$9 \mathrm{R}=\mathrm{OMe}$<smiles>CC(=O)C1=C2Nc3ccccc3[C@]23CCN2CC[C@]1(C)[C@H]2C3</smiles>

$13 \mathrm{~N}$-Oxyde<smiles>[R]OC(=O)C1(O)CC2(CC)C=CCN3CCc4c2ccc([R])cc2n1c4C23C(C)=O</smiles>

$15 \mathrm{R}=\mathrm{OMe}$

\subsection{Melodinus balansae Baill}

Venalstonine dehydro 14-15 (16), venalstonidine epoxy 14-15 $\alpha$ (17), paucivenine (18), pleiomutine (19) [34].

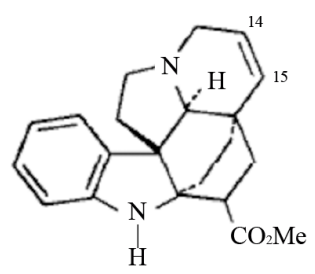

16 venalstonine déhydro $14-15$ 17 venalstonidine époxy $14-15 \alpha$

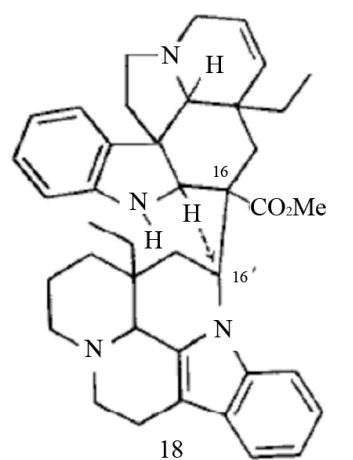

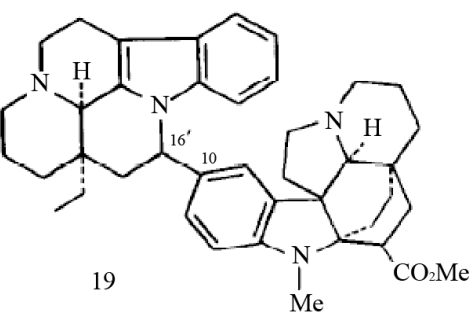




\subsection{Melodinus celastroides Baill}

(-) tabersoninel (20), leburnamine (21), lisoeburnamine (22), leburnamonine (23) [35].<smiles>CC[C@]12C=CCN3CC[C@@H]4Nc5ccccc5[C@H]4C(C(C)=O)=C(C1)[C@@H]32</smiles><smiles>[R]C1([R])CC2(CC)CCCN3CCc4c(n1c1ccccc41)C32</smiles>

$21 \quad \mathrm{R}_{1}=\mathrm{H}$ ax,; $\quad \mathrm{R}_{12}=\mathrm{OH}$ eg.

$22 \mathrm{R}_{1}=\mathrm{OH}$ ax.; $\mathrm{R}_{12}=\mathrm{OH}$ eg.

$\begin{array}{llll}23 & \mathrm{R}_{1} & \mathrm{R}_{2}=0\end{array}$

\subsection{Melodinus fusiformis Champ. ex Benth}

Melofusine I (24), melomorsine I (25), tabersonine (26), 11-hydroxytabersonine (27), 19-acetyltabersonine (28), 11-hydroxy-19-acetyl-tabersonine (29), vincadifformine (30), 11-hydroxyvincadifformine (31), vincadifformineNb-oxide (32), lochnericine (33), 11-hydroxy-14, 15-epoxytabersonine (34), venalstonine (35), venalstonidine (36), 19S-vindolinine (37), 19R-vindolinine (38), eburenine (39), (-)-aspidospermidine (40), (+)-voaphylline (41), (S)-quebrachamine (42), $N$-acyl-indolinique (43), scandine (44), 10-hydroxyscandine (45), meloscandonine (46), and 19-epi-meloscandonine (47) [20] [26] [30] [36]-[50].
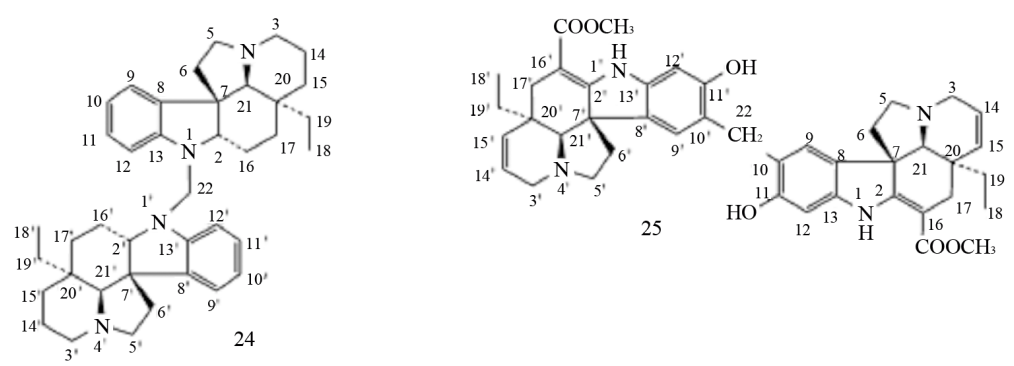
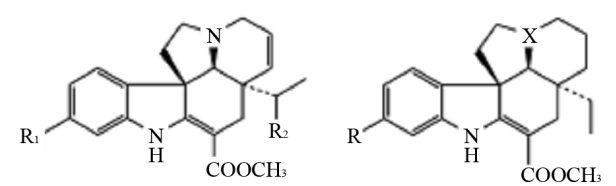

$$
\begin{array}{lll} 
& \mathrm{R}_{1} & \mathrm{R}_{2} \\
26 & \mathrm{H} & \mathrm{H} \\
27 & \mathrm{OH} & \mathrm{H} \\
28 & \mathrm{H} & \mathrm{Ac} \\
29 & \mathrm{OH} & \mathrm{Ac}
\end{array}
$$
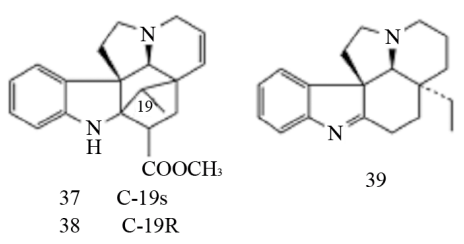

$$
39
$$$$
\begin{array}{ccc} 
& \mathrm{R} & \mathrm{X} \\
30 & \mathrm{H} & \mathrm{N} \\
31 & \mathrm{OH} & \mathrm{N} \\
32 & \mathrm{H} & \mathrm{N}=\mathrm{O}
\end{array}
$$

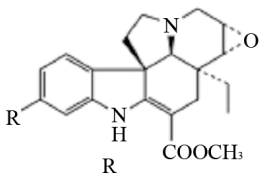

$\begin{array}{ll} & \mathrm{R} \\ 33 & \mathrm{H} \\ 34 & \mathrm{OH}\end{array}$

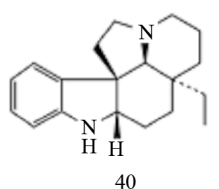

40

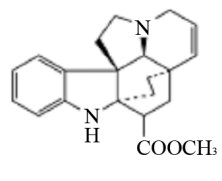

35

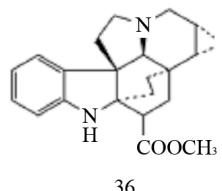

36

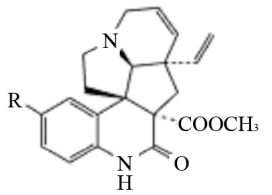

$\begin{array}{cc} & \mathrm{R} \\ 44 & \mathrm{H} \\ 45 & \mathrm{OH}\end{array}$

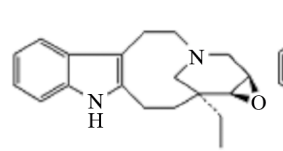

41

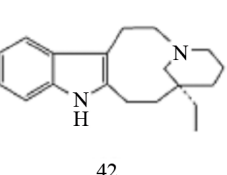

42

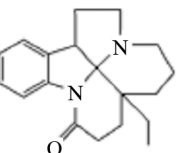

43

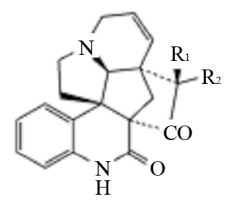

$\begin{array}{lll} & \mathrm{R} 1 & \mathrm{R} 2 \\ 46 & \mathrm{H} & \mathrm{CH}_{3}\end{array}$ 


\subsection{Melodinus guillauminii Boiteau}

11-hydroxytabersonme (48), venalstomne (49), venalstomdme (50), 14,15-seco-3-oxokopsmal (51), 3-oxovenalstomne (52), 11-methoxy- $\Delta^{14}$-vmcamenme (53), 3-0x0-hydroxykopsmme (54), ll-methoxyd14-vmcanol (55), kopsmme (56), 15-a-hydroxykopsmme (57), 16-epi- $\Delta^{14}$-vmcanol (58), $\Delta^{14}$-vmcanol (59), $\Delta^{14}$-vmcmone (60), $\Delta^{14}$-vmcamenme (61), $\Delta^{14}$-16-Eplvmcme (62), 11-methoxytabersonme (63), 19- $\beta$-hydroxyvenalstomne (64), plemcarpamme (65) [51].

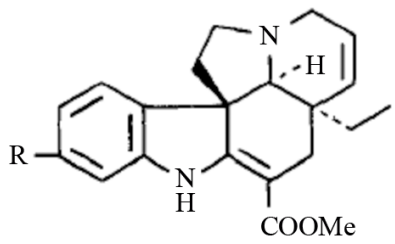

$$
\begin{array}{rl}
48 & \mathrm{R}=\mathrm{OH} \\
63 & \mathrm{R}=\mathrm{OMe}
\end{array}
$$

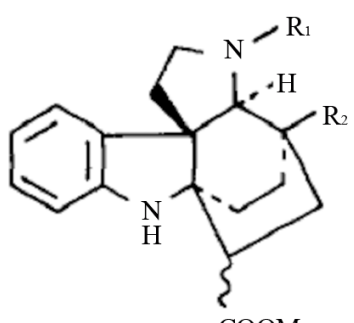

$\mathrm{COOMe}$

$51 \mathrm{R}_{1}=\mathrm{COMe}, \quad \mathrm{R}_{2}=\mathrm{CHO}$

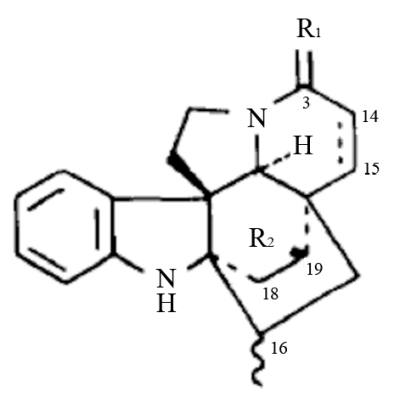

$\mathrm{COOMe}$

$49 \mathrm{R}_{1}=\mathrm{H}_{2}, \mathrm{R}_{2}=\mathrm{H}, \Delta^{14}, 16 \beta \mathrm{H}$

$50 \mathrm{R}_{1}=\mathrm{H}_{2}, \quad \mathrm{R}_{2}=\mathrm{H}, \quad 14 \alpha, \quad 15 \alpha$ epoxy, $16 \beta \mathrm{H}$

$52 \mathrm{R}_{1}=\mathrm{O}, \mathrm{R}_{2}=\mathrm{H}, \Delta^{14}, 16 \beta \mathrm{H}$

$54 \mathrm{R}_{1}=\mathrm{O}, \mathrm{R}_{2}=\mathrm{H},+\mathrm{OH}$ indetermined

$56 \mathrm{R}_{1}=\mathrm{H}_{2}, \quad \mathrm{R}_{2}=\mathrm{H}, 16 \beta \mathrm{H}$

$57 \mathrm{R}_{1}=\mathrm{H}_{2}, \quad \mathrm{R}_{2}=\mathrm{H}, 15 \alpha \mathrm{OH}, 16 \beta \mathrm{H}$

$64 \mathrm{R}_{1}=\mathrm{H}_{2} \mathrm{R}_{2}=\mathrm{OH}, \Delta^{14}, 16 \beta \mathrm{H}$

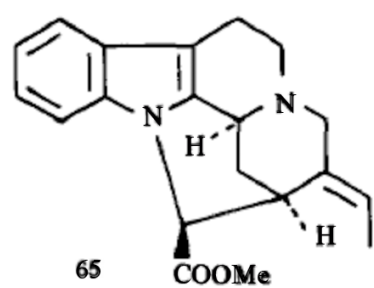

65

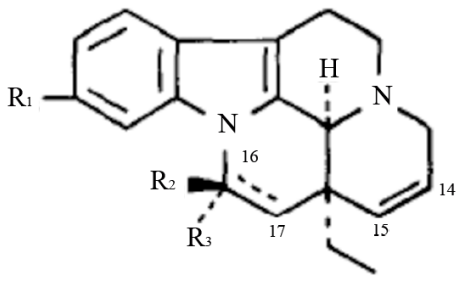

$53 \mathrm{R}_{1}=\mathrm{OMe}, \Delta^{16}$

$55 \mathrm{R}_{1}=\mathrm{OMe}, \mathrm{R}_{2}=\mathrm{H}, \mathrm{R}_{3}=\mathrm{OH}$

$58 \mathrm{R}_{1}=\mathrm{H}, \mathrm{R}_{2}=\mathrm{OH}, \mathrm{R}_{3}=\mathrm{H}$

$59 \mathrm{R}_{1}=\mathrm{H}, \mathrm{R}_{2}=\mathrm{H}, \mathrm{R}_{3}=\mathrm{OH}$

$60 \mathrm{R}_{1}=\mathrm{OMe}, \mathrm{R}$

$$
\mathrm{R}_{3}>=\mathrm{O}
$$

$61 \mathrm{R}_{1}=\mathrm{H}, \Delta^{16}$

$62 \mathrm{R}_{1}=\mathrm{OMe}, \mathrm{R}_{2}=\mathrm{COOMe}, \mathrm{R}_{3}=\mathrm{OH}$

\subsection{Melodinus hemsleyanus Diels}

Tabersonine (66), 11-methoxytabersonine (67), 11-hydroxytabersonine (68), 11,19R-dihydroxytabersonine (69), ll-hydroxy-14,15- $\alpha$-epoxytabersonine (70), scandine (71), 10-hydroxyscandine (72), meloscine (73), meloscandonine (74), venalstonine (75), venalstonidine (76), vindolinine (77), picralinal (78), picrinine (79), akuammidine (80), ll-hydroxyvincadifformine (81), 14,15-epoxyscandine (82), 19-epimeloscandonine (83), 16 $\beta$-hydroxy-19R-vindolinine (84) and 16 $\beta$-hydroxy-19S-vindolinine (85) [39].<smiles></smiles>

75<smiles>CC(=O)C1CC23CC1C14CCN(CC5OC51)N2CCC43</smiles>

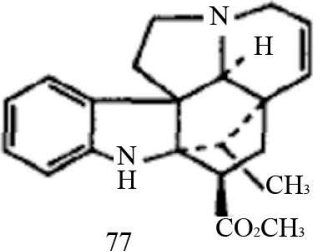

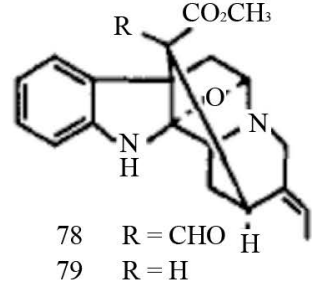




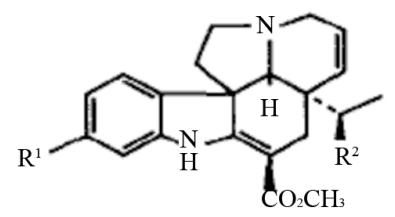

$\begin{array}{lll} & \mathrm{R}^{1} & \mathrm{R}^{2} \\ 66 & \mathrm{H} & \mathrm{H} \\ 67 & \mathrm{OCH}_{3} & \mathrm{H} \\ 68 & \mathrm{OH} & \mathrm{H} \\ 69 & \mathrm{OH} & \mathrm{OH}\end{array}$

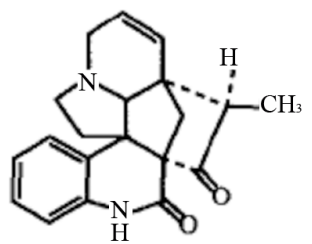

74

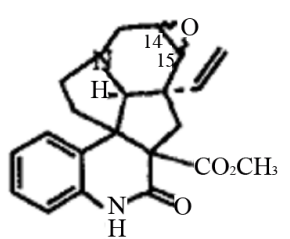

82<smiles>CCC12CC(C(C)=O)=C3Nc4cc(O)ccc4C34CCN(CC3OC31)C42</smiles>

70

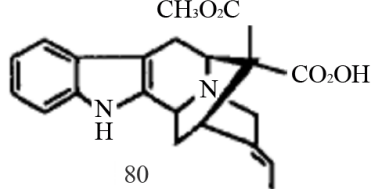

80

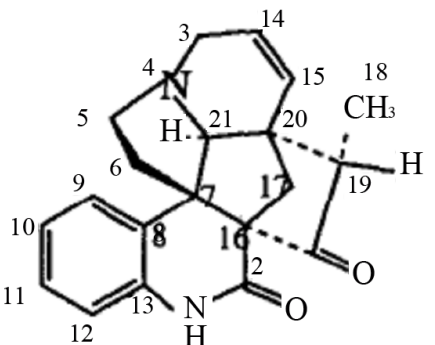

83<smiles>[R]c1ccc2c(c1)C13CCN4CC=CC(C=C)(C1)C4([R])C(=O)NC23</smiles><smiles>[R]C(=O)OC</smiles><smiles>CCC12CCCN3CC[C@@]4(Nc5cc(O)ccc54)C(C(=O)OC)=C(C1)C32</smiles>

$\begin{array}{ll}84 & 19 \mathrm{R} \\ 85 & 19 \mathrm{~S}\end{array}$

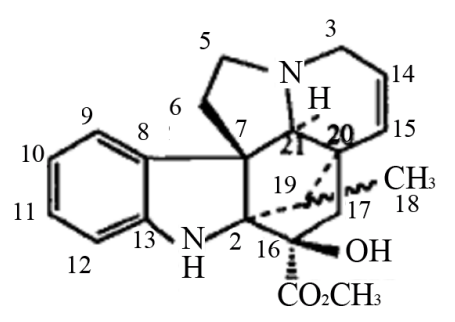

\subsection{Melodinus morsei Tsiang}

Melofusine I (24), melomorsine I (25), tabersonine (26), 11-hydroxytabersonine (27), 19-acetyltabersonine (28), 11-hydroxy-19-acetyl-tabersonine (29), vincadifformine (30), 11-hydroxyvincadifformine (31), vincadifformineNb-oxide (32), lochnericine (33), 11-hydroxy-14,15-epoxytabersonine (34), venalstonine (35), venalstonidine (36), 19S-vindolinine (37), 19R-vindolinine (38), eburenine (39), (-)-aspidospermidine (40), (+)-voaphylline (41), (S)-quebrachamine (42), $N$-acyl-indolinique (43), scandine (44), 10-hydroxyscandine (45), meloscandonine (46), and 19-epi-meloscandonine (47) [17] [20] [26] [30] [36]-[50] [52].

\subsection{Melodinus monogynus Roxb}

Medigenin (86), Medinin (87), medigenin acetate (88) [53].
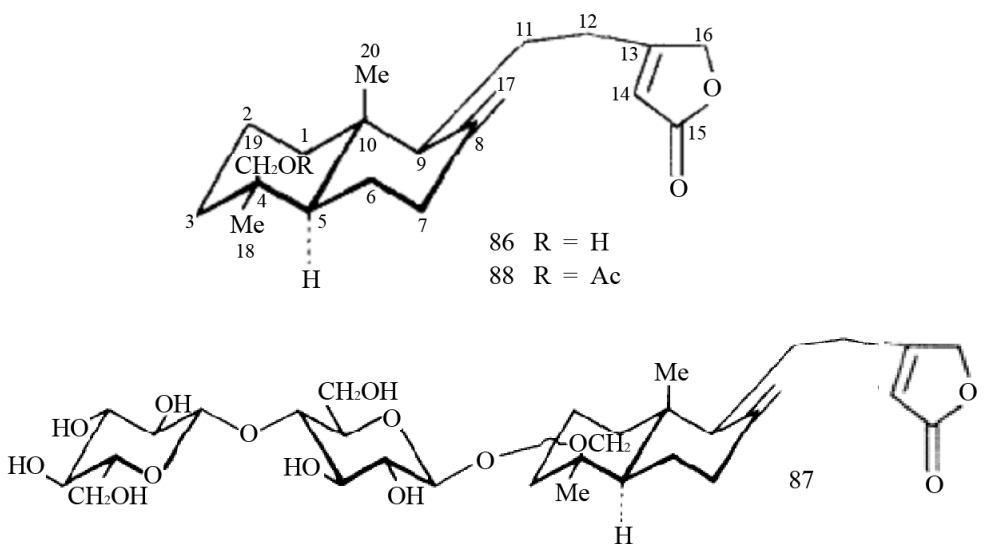


\subsection{Melodinus scandens J.R. Forst. \& G. Forst.}

9-hydroxyepimeloscine (89); epimeloscine (90), 9-methoxyepimeloscine (91) [27].

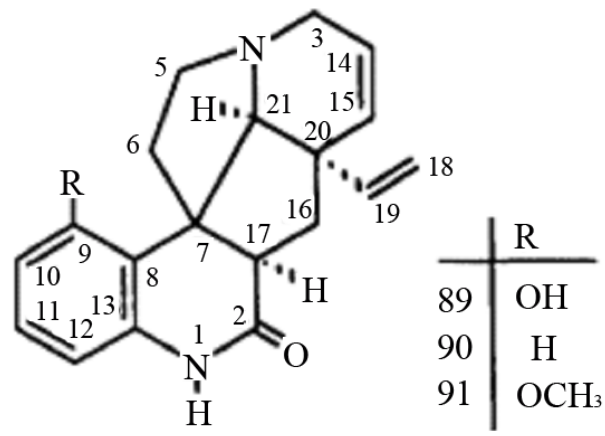

\subsection{Melodinus suaveolens (Hance) Champ. ex Benth}

Melodinine M (92), Melodinine N (93), Melodinine O (94), Melodinine P (95), Melodinine Q (96), Melodinine R (97), Melodinine S (98), Melodinine T (99), Melodinine U (100), 11-hydroxytabersonine (27), 11-methoxytabersonine (67), tabersonine (26), 19-(R)-acetoxytabersonine (101), 11-methoxy-19-(R)-hydroxytabersonine (102), lochnericine (33), $\Delta^{14}$-vincine (103), vindolinine (77), 11-methoxytabersonine (67), vincadifformine (30), hazuntine (104), 11-hydroxytabersonine (27). 11-methoxyvincadifformine (105), cathovalinine (106), vincoline (107), 19R-hydroxytabersonine (108), 1l-methoxy-19R-hydroxytabersonine (109), 11,19R-dihydroxytabersonine (110), tabersonine (26), suaveolenine (111) [25] [26] [54].

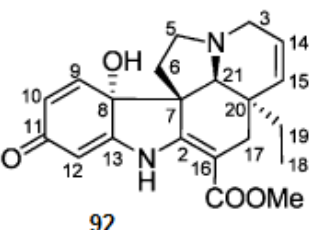

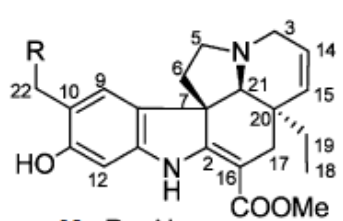

$93 \mathrm{R}=\mathrm{H}$

$94 \mathrm{R}=\mathrm{OMe}$<smiles>COC(=O)C1=C[C@@H]2C=C/C(=C\C(C)=O)N3CC[C@@]4(c5ccccc5N[C@@]124)[C@@H]3O</smiles>

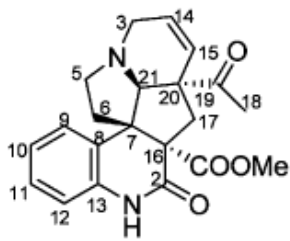

99<smiles>[R]c1cc2c(cc1[R])C1=C(C(=O)OC)CC3(C([R3])C)CCCN4CCC3(N2)C14</smiles>

$\begin{array}{lll}R_{1} & R_{2} & R_{3}\end{array}$

$95 \mathrm{OH} \quad \mathrm{H} \quad \mathrm{H} \quad \Delta^{14,15}$

$101 \mathrm{H} \quad \mathrm{H}$ OAC $\Delta^{14,15}$

$102 \mathrm{H} \quad \mathrm{OMe} \mathrm{OH} \quad \Delta^{14,15}$<smiles>[R]C(C)[C@]12CC[C@H](CC(C)=O)N3CC[C@]4(C(=C(C(=O)OC)C1)Nc1ccccc14)[C@@H]32</smiles>

$97 \mathrm{R}=\mathrm{OH}, \Delta^{14,15}$

$98 \mathrm{R}=\mathrm{H}, \quad 14,15-\alpha$-ероху $103 \mathrm{R}=\mathrm{H}, \Delta^{14,15}$

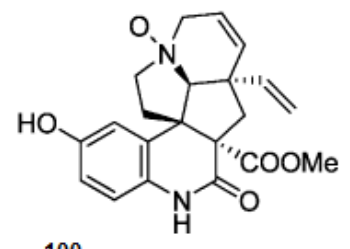

100<smiles>CCC12C=CCN3CCc4c(n1c1cc(OC)ccc41)[C@](O)(C(C)=O)C[C@]32C</smiles>

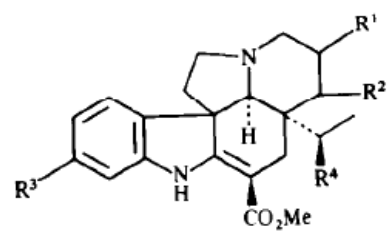

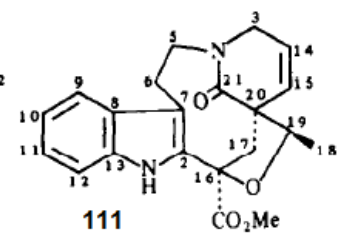<smiles>[R]C(OC)[R]([R])([R])OC</smiles><smiles>[R]C(=O)OC([R])(O)C([R])O</smiles>

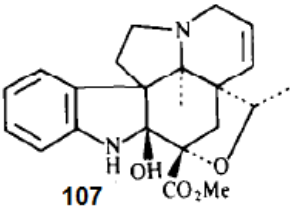

$110 \mathrm{OH} \quad \mathrm{OH}$ 


\subsection{Melodinus yunnanensis Tsiang \& P.T. Li}

Meloyine I (112), 19S-Methoxytubotaiwine $\mathrm{N}_{4}$-oxide (113), 16,19-Epoxy- $\Delta^{14}$-vincanol (114), 14 $\beta$-Hydroxymeloyunine (115), Meloyunine (116), $\Delta^{14}$-Vincamenine $\mathrm{N}_{4}$-oxide (117), 16 $\beta, 21 \beta$-Epoxy-vincadine (118), 14 $\beta, 15 \beta$-20S-Quebrachamine (119), 3-Oxo-voaphylline (120), $2 \alpha, 7 \alpha$-Dihydroxy-dihydrovoaphylline (121) [16] [17] [55].
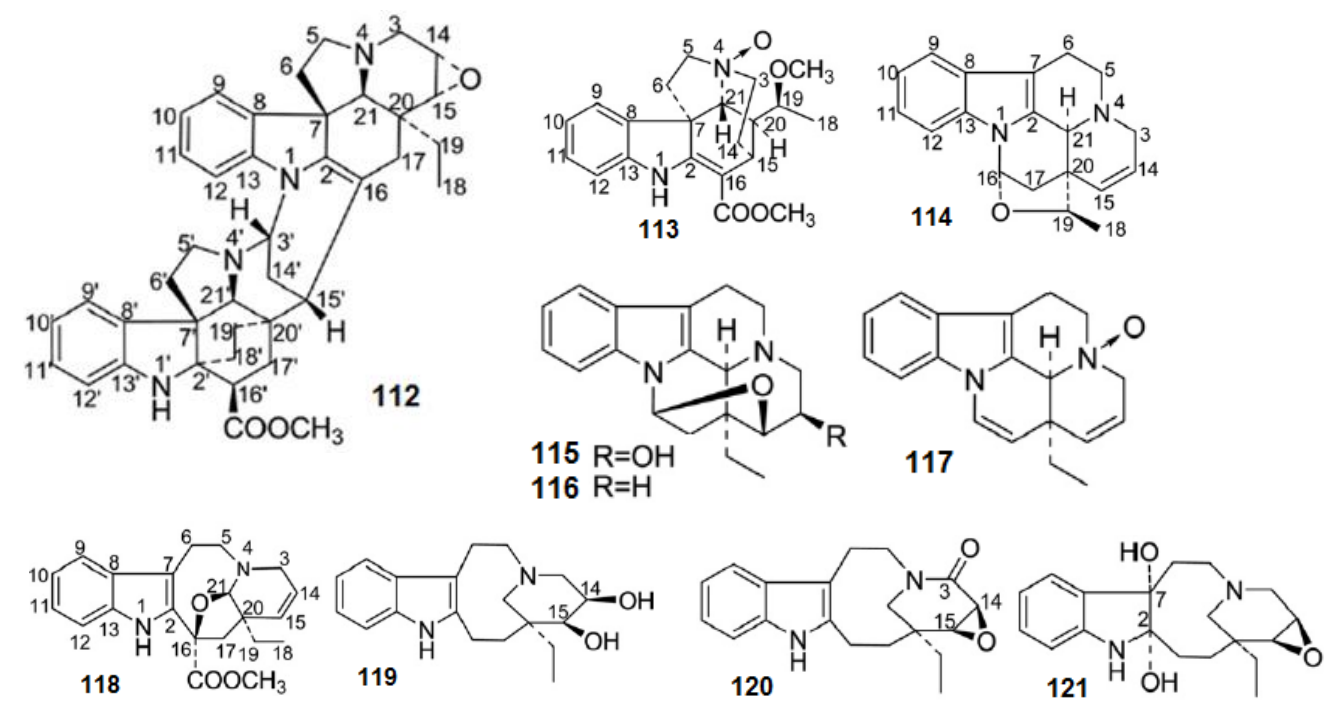

\section{Biological Activities}

In vitro pharmacological activities of Melodinus.

Four species from Melodinus have been studied for their pharmacological activities. Extracts and pure compounds derived from Melodinus were reported to have a concentrate pharmacological activity of antitumor.

\subsection{Anticancer Activities}

It is known that nature is able to produce a wide variety of chemical entities of novel structure. Many of the new and novel compounds isolated from natural sources might otherwise have never been discovered, especially those of considerable complexity requiring the development of methods for the creation of new ring systems. Natural products appeared to be a promising source for new types of compounds with antitumor activity [56] [57].

MTT assay is dependent on the reduction of the tetrazolium salt MTT (3-(4,5-dimethylthazol-2-yl)-2,5-diphenyl tetrazolium bromide) by the mitochondrial dehydrogenase of viable cells to form a blue formazan product. The assay measures cell respiration and the amount of formazan produced is proportional to the number of living cells present in culture which will result in lower optical density (OD) [58].

Anticancer activities were reported in 1998, Demethyltenuicausine (I), a new bisindole alkaloid, was isolated from Melodinus hemsleyanus revealed antitumor activities in pharmacological tests [24].

Antitumor potential was demonstrated by the indole alkaloids from the leaves and twigs of Melodinus fusiformis and Melodinus tenuicaudatus via the MTT biological assay showed significant cytotoxicity against five human tumor cell lines: SW480, SMMC-7721, HL-60, MCF-7 and A-549 [17] [18].

Nine isolated from Melodinus suaveolens revealed a positive cytotoxicity with $\mathrm{IC}_{50}$ all around $10 \mu \mathrm{M}$ against five human cancer cell lines: Five human cancer cell lines, human myeloid leukemia HL-60, hepatocellular carcinoma SMMC-7721, lung cancer A-549, breast cancer MCF-7, and colon cancer SW480 cells [25].

\subsection{Other Biological Activities}

A pharmacological test revealed that 11-hydroxyvincadifformine which was isolated from the aerial parts of Melodinus hemsleyanus has significant antifertility activity [19]. 


\section{Future Perspectives and Conclusions}

There is no relative report mentioned the Melodinus products. However, according to its potential, Melodinus, can be published as a high-value export crop for medicinal use.

Although, several research work has been done on some plants of this genus to date, but a large number of species are still chemically and/or pharmacologically unknown. Consequently, a broad field of future research remains possible in which the isolation of new active principles from these species would be of great scientific merit. The alkaloids are of particular interest as many are highly potent bioactive and perhaps responsible for most of activities shown by the plants of this genus. However, the mechanism of their action is still unknown. Hence, a detailed study is required to understand the structure-activity relationship of these constituents. As literature showed, many plant extracts having cytotoxic activity, hence, the particular constituent responsible for the activity may be isolated for further process. In addition, some plant extracts were only screened for their preliminary in vitro activities, so, the advance clinical trial of them deserves to be further investigated. Herein, we described the possible applications in clinical research but further investigations on phytochemical discovery and subsequent screening are needed for opening new opportunities to develop pharmaceuticals based on Stephania constituents.

\section{Acknowledgements}

This work was supported by University of Nottingham, Malaysia Campus under the $\mathrm{PhD}$ research grant. The authors pay their sincere thanks to editor and referees of the journal, for their valuable suggestions to improve this article. There is no conflict of interest for all authors.

\section{References}

[1] Endress, M.E. and Bruyns, P.V. (2000) A Revised Classification of the Apocynaceae s.l. The Botanical Review, 66, 1-56. http://dx.doi.org/10.1007/BF02857781

[2] Angiosperm Phylogeny Group (2009) An Update of the Angiosperm Phylogeny Group Classification for the Orders and Families of Flowering Plants: APG III. Botanical Journal of the Linnean Society, 161, 105-121. http://dx.doi.org/10.1111/j.1095-8339.2009.00996.x

[3] Phillipson, J.D. (2007) Phytochemistry and Pharmacognosy. Phytochemistry, 68, 2960-2972. http://dx.doi.org/10.1016/j.phytochem.2007.06.028

[4] Fabricant, D.S.F.N. (2001) The Value of Plants Used in Traditional Medicine for Drug Discovery. Environmental Health Perspectives, 109, 69-75. http://dx.doi.org/10.1289/ehp.01109s169

[5] Soejarto, D.D. (2005) Ethnographic Component and Organism Documentation in an Ethnopharmacology Paper: A “Minimum” Standard. Journal of Ethnopharmacology, 100, 27-29. http://dx.doi.org/10.1016/j.jep.2005.06.005

[6] Rapanarivo, S.H.J.V. and Leeuwenberg, A.J.M. (1999) Taxonomic revision of Pachypodium. Series of Revisions of Apocynaceac XLVIII. In: Rapanarivo, S.H.J.V., Ed., Pachypodium (Apocynaceae): Taxonomy, Habitats and Cultivation, Balkema, 1-82.

[7] (2012) Saba Comorensis in Agroforestree Database; World Checklist of Seed Plants. International Association for Plant Taxonomy.

[8] (2013) Melodinus. The Plant List; World Checklist of Seed Plants. International Association for Plant Taxonomy.

[9] Stickman (1972) Tree Flora of Malaya. Journal of Natural Products, 1, 126-127.

[10] Wiart, C. (2006) Medicinal Plant of Asia-Pacific-Drugs for the Future. World Scientific Publishing Co. Pte. Ltd., British Library Cataloguing-in-Publication Data, 380.

[11] (1977) Delectis Florae Reipublicae Popularis Sinicae Agendae, Florae Reipublicae Popularis Sinicae. Academiae Sinicae Edita, 63, 24.

[12] Au, K. S. and Cray, D. E. (1969) A New Antibiotic? Biochemical Pharmacology, 18, 2673. http://dx.doi.org/10.1016/0006-2952(69)90198-1

[13] Lam, K.S. (2007) New Aspects of Natural Products in Drug Discovery. Trends in Microbiology, 15, 279-289. http://dx.doi.org/10.1016/j.tim.2007.04.001

[14] Hu, W. L. and Hesse, M. (1988) The Alkaloids of Melodinus Acutiflorus. Rhyzicine N-Oxide. Plantamedica, 54, 235236. http://dx.doi.org/10.1055/s-2006-962414

[15] Y L Zhou, J H Ye, Z M Li, Z H Huang (1988) Study on the Alkaloids of Melodinus Tenuicaudatus. Plantamedica, 54, 315-317. http://dx.doi.org/10.1055/s-2006-962443 
[16] Feng, T., Cai, X.H., Li, Y., Wang, Y.Y., Liu, Y.P., Xie, M.J. and Luo, X.D. (2009) Melohenines A and B, Two Unprecedented Alkaloids from Melodinus henryi. Organic Letters, 11, 4834-4837. http://dx.doi.org/10.1021/ol9018826

[17] Cai, X.H., Jiang, H., Li, Y., Cheng, G.G., Liu, Y.P., Feng, T. and Luo, X.D. (2011) Cytotoxic Indole Alkaloids from Melodinus fusiformis and Melodinus morsei. Chinese Journal of Natural Medicines, 9, 259-263.

[18] Feng, T., Li, Y., Wang, Y.Y., Cai, X.H., Liu, Y.P. and Luo, X.D. (2010) Cytotoxic Indole Alkaloids from Melodinus tenuicaudatus. Journal of Natural Products, 73, 1075-1079. http://dx.doi.org/10.1021/np100086x

[19] Guo, L.W. and Zhou, Y.L. (1993) Alkaloids from Melodinus hemsleyanus. Phytochemistry, 34, 563-566. http://dx.doi.org/10.1016/0031-9422(93)80046-U

[20] He, Y.L., Chen, W.M. and Feng, X.Z. (1994) The Alkaloids of Melodinus morsei. Phytochemistry, 37, 1055-1057. http://dx.doi.org/10.1016/S0031-9422(00)89527-8

[21] Goldberg, A.F.G. and Stoltz, B.M. (2011) A Palladium-Catalyzed Vinylcyclopropane (3 + 2) Cycloaddition Approach to the Melodinus Alkaloids. Organic Letters, 13, 4474-4476. http://dx.doi.org/10.1021/ol2017615

[22] Feldman, K.S. and Antoline, J.F. (2013) Synthesis Studies on the Melodinus Alkaloid Meloscine. Tetrahedron, 69, 1434-1445. http://dx.doi.org/10.1016/j.tet.2012.12.032

[23] Abe, F. and Yamauchi, T. (1993) Indole Alkaloids from Leaves and Stems of Leuconotis eugenifolius. Phytochemistry, 35, 169-171. http://dx.doi.org/10.1016/S0031-9422(00)90527-2

[24] Yan, K., Hong, S. and Feng, X. (1998) Demethyltenuicausine, a New Bisindole Alkaloid from Melodinus hemsleyanus. Acta Pharmaceutica Sinica, 33, 597-599.

[25] Cai, X.H., Li, Y., Li, X.N., Luo, X.D., Bao, M.F. and Liu, Y.P. (2012) Alkaloids from Melodinus yunnanensis. Phytochemistry, 83, 116-124. http://dx.doi.org/10.1016/j.phytochem.2012.06.013

[26] Jian, H.Y., Zhou, Y.L., Huang, Z.H., et al. (1991) Alkaloids from Melodinus suaveolens. Phytochemistry, 30, 31683170. http://dx.doi.org/10.1016/S0031-9422(00)98284-0

[27] Plat, M., Mehri, H. and Diallo, A.O. (1995) An Alkaloid from Leaves of Melodinus scandens. Phytochemistry, 40, 1005-1006. http://dx.doi.org/10.1016/0031-9422(95)00334-4

[28] Feng, T., Li, Y., Liu, Y.P., Cai, X.H., Wang, Y.Y. and Luo, X.D. (2010) Melotenine A, a Cytotoxic Monoterpenoid Indole Alkaloid from Melodinus tenuicaudatus. Organic Letters, 12, 968-971. http://dx.doi.org/10.1021/ol1000022

[29] Feng, T., Cai, X.H., Liu, Y.P., Li, Y., Wang, Y.Y. and Luo, X.D. (2010) Melodinines A-G, Monoterpenoid Indole Alkaloids from Melodinus henryi. Journal of Natural Products, 73, 22-26. http://dx.doi.org/10.1021/np900595v

[30] He, X., Zhou, Y.L. and Huang, Z.H. (1992) Study on the Alkaloids of Melodinus fusiformis. Acta Chimica Sinica, 50, 96-101.

[31] Handa, S.S. (2007) Status of Medicinal and Aromatic Plants (MAPs) Utilization Globally Including Issues of Quality Control and Technologies for Large Scale Production of Plant Based Products. United Nations Industrial Development Organization (UNIDO).

[32] Hu, W.L., Zhu, J.P., AkiinI, U., Prewo, R. and Hesse, M. (1987) Revision of the Structures of Rhazicine and Rhazimine, Two Alkaloids from Melodinus acutiflorus. Phytochemistry, 26, 2625-2630.

[33] Baassou, S., Mehri, H. and Plat, M. (1978) Alkaloids of Melodinus aeneus. Phytochemistry, 17, 1449-1450. http://dx.doi.org/10.1016/S0031-9422(00)94618-1

[34] Mehri, M.H., et al. (1978) Alkaloids of Melodinus Balansae var Paucivenosus. Phytochemistry, 17, 1451-1452.

[35] Rabaron, A. and Plat, M. (1973) Isolation of the Dehydro-14 Isoeburnamine the Melodinus celastroides. Phytochemistry, 12, 2537-2539. http://dx.doi.org/10.1016/0031-9422(73)80473-X

[36] Ziegler, F.E. and Bennett, G.B. (1971) Total Synthesis of (+ -)-Tabersonine. Journal of the American Chemical Society, 93, 5930-5931. http://dx.doi.org/10.1021/ja00751a076

[37] Kutney, J.P., Choi, L.S.L., Kolodziejczy, P., et al. (1980) Alkaloid Production in Catharanthus roseus Cell Cultures: Isolation and Characterization of Alkaloids from One Cell Line. Phytochemistry, 19, 2589-2595. http://dx.doi.org/10.1016/S0031-9422(00)83924-2

[38] Kobayashi, S., Peng, G. and Fukuyama, T. (1999) Efficient Total Syntheses of ( \pm )-Vincadifformine and Tabersonine. Tetrahedron Letters, 40, 1519-1522. http://dx.doi.org/10.1016/S0040-4039(98)02667-7

[39] Guo, L.W. and Zhou, Y.L. (1993) Alkaloids from Melodinus hemsleyanus. Phytochemistry, 34, 563-566. http://dx.doi.org/10.1016/0031-9422(93)80046-U

[40] Aimi, N., Asada, Y., Sakai, S. and Haginiwa, J. (1978) Studies on Plants Containing Indole Alkaloids. VII. Isolation of Several Aspidosperma- and Vincamine-Type Alkaloids from the Seeds of Amsonia elliptica ROEM-Schult. Chemical \& Pharmaceutical Bulletin, 26, 1182-1187. http://dx.doi.org/10.1248/cpb.26.1182

[41] Moza, B.K., Das, K.G., Trojanek, J., et al. (1964) Chemistry of Lochnericine. Lloydia, 27, 267. 
[42] Ahond, A., Janot, M.M., Langlois, N., Lukacs, G., Potier, P., Rasoanaivo, P., Sangare, M., Neuss, N. and Plat, M. (1974) Structure of Vindolinine. Journal of the American Chemical Society, 96, 633-634. http://dx.doi.org/10.1021/ja00809a078

[43] Olivier, L., Quirin, F., Mauperin, P., et al. (1970) Structure of Eburenine Alkaloids from Seeds of Huneria Eburneapichon (Apocynaceae). Comptes Rendus Hebdomadaires des Seances de L'Academie des Sciences Serie C, 270, 1667.

[44] Ihara, M., Yasui, K., Taniguchi, N., et al. (1990) An Asymmetric-Systhesis of a Quaternary Chiral Building Block from Tehylmalonic Acid-A Preparation of Key Synthetic Intermediates of Hunteria-Type and Aspidosperma-Type Indole Alkaloids. Heterocycles, 31, 1017-1020. http://dx.doi.org/10.3987/COM-90-5418

[45] Kam, T.S. and Anuradha, S. (1995) Alkaloids from Tabernaemontana divaricate. Phytochemistry, 40, 313-316. http://dx.doi.org/10.1016/0031-9422(95)00266-A

[46] Asaoka, M. and Takei, H. (1989) A New Enantioselectlve Route to (+)-uebrachamine. Heterocycles, 29, $243-244$. http://dx.doi.org/10.3987/COM-89-4810

[47] Hugel, G., Levy, G. and Le Men, J. (1974) Methylene-indoles, indolenines et indoleniniums, IX nouveau rearrangement de la dehydro-1,2 Aspidospermidine. Tetrahedron Letters, 15, 3109-3112. http://dx.doi.org/10.1016/S0040-4039(01)91835-0

[48] Daudon, M., Mehri, M.H., Plat, M.M., Hagaman, E.W., Schell, F.M. and Wenkert, E. (1975) Carbon-13 Nuclear Magnetic Resonance Spectroscopy of Naturally Occurring Substances. XXXIV. Monomeric Quinolinic Melodinus Alkaloids. Journal of Organic Chemistry, 40, 2838-2839. http://dx.doi.org/10.1021/jo00907a031

[49] Li, C.M., Zheng, H.L., Wu, S.G., et al. (1994) Quinolinicmelodinus Alkaloids from Stem Bark Melodinus khasianus. Acta Botanic Yunnanica, 16, 1-3.

[50] Suau, R., Silva, L.V. and Valpuesta, M. (1993) Quaternary Protoberberine Alkaloids from Ceratocapnos heterocarpa. Phytochemistry, 34, 559-561. http://dx.doi.org/10.1016/0031-9422(93)80045-T

[51] Zeches, M., et al. (1984) Alkaloids of Melodinus guillauminii. Phytochemistry, 23, 171-174. http://dx.doi.org/10.1016/0031-9422(84)83101-5

[52] He, Y.L., Chen, W.M. and Feng, X.Z. (1994) Melomorsine, a New Dimeric Indoline Alkaloid from Melodinus morsei. Journal of Natural Products, 57, 411-414. http://dx.doi.org/10.1021/np50105a016

[53] Sethi, A., et al. (1988) A Labdane Diterpene and Its Glycoside from Melodinus monogynus. Phytochemistry, 27, 22552259. http://dx.doi.org/10.1016/0031-9422(88)80137-7

[54] Lai, M.C.S., Au, K.S. and Gray, D.E. (1969) Alkaloids of Melodinus suaveolens and Their Excretion as a Common End-Product in the Rat. Biochemical Pharmacology, 18, 1553-1557. http://dx.doi.org/10.1016/0006-2952(69)90142-7

[55] Liu, Y.P., Li, Y., Cai, X.H., Li, X.Y., Kong, L.M., Cheng, G.G. and Luo, X.D. (2012) Melodinines M-U, Cytotoxic Alkaloids from Melodinus suaveolens. Journal of Natural Products, 75, 220-224. http://dx.doi.org/10.1021/np2009169

[56] Hartwell, J. (1976) Types of Anticancer Agents Isolated From Plants. Cancer Treatment Reports 60.

[57] Mosmann, T. (1983) Rapid Colorimetric Assay for Cellular Growth and Survival: Application to Proliferation and Cytotoxicity Assays. Journal of Immunological Methods, 65, 55-63.

[58] Mortimer, C., Wells, G., Crochard, J.P., Stone, E.L., Bradshaw, T.D., Stevens, M.F.G. and Westwell, A.D. (2005) Antitumor Benzothiazoles. 26.1 2-(3,4-Dimethoxyphenyl)-5-fluorobenzothiazole (GW 610, NSC 721648), a Simple Fluorinated 2-Arylbenzothiazole, Shows Potent and Selective Inhibitory Activity against Lung, Colon, and Breast Cancer Cell Lines. Journal of Medicinal Chemistry, 49, 179-185. 